\title{
Combining clinical examination with exome sequencing for the diagnosis and treatment of Marfan syndrome: a case series of 6 families from China
}

\author{
Yanyu Duan ${ }^{1,2,3}$, Peijun $\mathrm{Li}^{2}$, Ting Ding ${ }^{2}$, Yabin Wang ${ }^{1}$, Yongling $\mathrm{Liao}^{2}$, Zhiming $\mathrm{Du}^{2}$, Jiayuan Ling ${ }^{2}$, \\ Shaoqiang $\mathrm{Liu}^{4}$, Weisong $\mathrm{Li}^{5}$, Ziyou Liu ${ }^{1,2,3}$ \\ ${ }^{1}$ Engineering Research Center of Intelligent Acoustic Signals of Jiangxi Province, Key Laboratory of Prevention and Treatment of Cardiovascular \\ and Cerebrovascular Diseases, Ministry of Education, Gannan Medical University, Ganzhou, China; ${ }^{2}$ Heart Medical Centre, First Affiliated Hospital \\ of Gannan Medical University, Ganzhou, China; ${ }^{3}$ Ganzhou Cardiovascular Rare Disease Diagnosis and Treatment Technology Innovation Center, \\ Gannan Medical University, Ganzhou, China; ${ }^{4}$ Radiology Department, First Affiliated Hospital of Gannan Medical University, Ganzhou, China; \\ ${ }^{5}$ Department of Pathology, First Affiliated Hospital of Gannan Medical University, Ganzhou, China \\ Contributions: (I) Conception and design: Y Duan; (II) Administrative support: Y Duan, Z Liu; (III) Provision of study materials or patients: Z Liu, Y \\ Liao, Z Du, J Ling, S Liu, W Li, P Li; (IV) Collection and assembly of data: Y Duan, T Ding, Y Wang; (V) Data analysis and interpretation: Z Liu, \\ Y Duan; (VI) Manuscript writing: All authors; (VII) Final approval of manuscript: All authors. \\ Correspondence to: Ziyou Liu. Engineering Research Center of Intelligent Acoustic Signals of Jiangxi Province, Key Laboratory of Prevention \\ and Treatment of Cardiovascular and Cerebrovascular Diseases, Ministry of Education, Gannan Medical University, Ganzhou 341000, China. \\ Email: ziyoudoc@126.com.
}

Background: Marfan syndrome (MFS) is a rare autosomal dominant connective tissue disorder. Diagnosing MFS can be challenging as the disease's severity and clinical manifestations differ between pathogenic variants, and because a lack of published information currently exists on phenotype-genotype correlations. This report aims to underline the clinical manifestations associated with fibrillin-1 (FBN1) gene mutations by assessing MFS in 6 families from China.

Methods: We diagnosed 6 patients and their relatives with MFS by combining a clinical examination (based on the 2010 revised Ghent nosology criteria) with a targeted next-generation sequencing analysis. The functional analysis of the causal mutations and clinical details of the affected patients were then assessed.

Results: We identified 6 pathogenic mutations in FBN1, including 1 novel frameshift, 1 nonsense, and 4 missense mutations. Most uniquely, mitral valve prolapses (MVP) and ectopia lentis (EL) were found in the cysteine-related mutations. Typically, facial symptoms of MFS are observed in frameshift or nonsense mutants, not in cysteine-related ones. Furthermore, the patients with premature terminal codons had a more serious skin condition than patients with missense mutations, partly indicating the important effect FBN1 has on skin.

Conclusions: This study expands the mutation spectrum of MFS and highlights possible genotypephenotype correlations, thereby improving the early diagnosis and symptomatic treatment of the disease.

Keywords: Marfan syndrome (MFS); fibrillin-1 (FBN1); phenotypic variability; genotype-phenotype correlation; case series

Submitted Jul 30, 2021. Accepted for publication Sep 09, 2021.

doi: 10.21037/apm-21-2305

View this article at: https://dx.doi.org/10.21037/apm-21-2305 


\section{Introduction}

Marfan syndrome (MFS; OMIM\#154700) is an inherited connective tissue disorder that affects approximately 1 in 5,000 people $(1,2)$. This autosomal dominant syndrome is mainly characterized by a combination of cardiovascular, ocular, skeletal, pulmonary, skin, and dural anomalies, reflecting the highly variable clinical manifestations of the disease. In addition, the MFS phenotype gradually evolves with an increase in age, making its diagnosis in children and some adults particularly challenging. Because of these difficulties, the 2010 revised Ghent nosology criteria now considers causative gene mutations as a major diagnostic criterion (3).

More than $90 \%$ of MFS cases are characterized by defects in the fibrillin-1 (FBN1) gene (4), while a minority of cases are associated with the transforming growth factor beta receptor II (TGFBR2) gene (5). FBN1 is a $350-\mathrm{kD}$ extracellular matrix glycoprotein that has a modular structure with 47 repeats of epidermal growth factor (EGF)like domains, 7 TGF $\beta$-binding (TB) protein-like domains, and 1 heterozygous domain. The EGF-like domains contain a six-cysteine motif and serve the calcium-binding microfibrillar structure, while the TB-like domains contain an eight-cysteine motif and serve the TGF $\beta$ extracellular microfibrillar structure (6). For this reason, FBN1 plays a pivotal role in structural integrity and as a mediator of TGF $\beta$ signaling (7).

Most pathogenic mutations are unique to individual MFS families, and $25 \%$ of MFS cases display de novo mutations (8). To date, more than 3,000 pathogenic mutations have been identified in FBN1. These mutations have mainly been classified into 2 major mutation categories: haploinsufficient (HI) mutations (resulting from a deficient $F B N 1$ protein) and dominant-negative (DN) mutations (resulting in an abnormal FBN1 formation) (9). Several studies have demonstrated a higher probability of aortic surgery and cardiovascular death in MFS patients with HI mutations in FBN1 (10), while DN mutations in exons 24-32 are predictive of neonatal MFS (even in non-neonatal cases), which is a severe cardiovascular phenotype (11). More recently, genotype-phenotype correlations have been recognized as an important factor not only in the diagnosis of MFS, but also in risk stratification and clinical decisions related to the disease (12). To date, the correlation reports have been focused on the unrelated individuals in cohort study, not focused enough on phenotypic variation between family members carried the same mutation. It is necessary to further accumulate clinical family cases and improve the overall understanding of the pathogenesis of the disease. In this study, we described the diagnosis for 6 MFS families from China combining clinical examination with exome sequencing, 6 pathogenic mutations in FBN1 were identified including a novel HI mutation. Possible phenotype-genotype correlations were also analyzed with the hope that it might improve the precision of treating and managing suspected MFS cases. We present the following article in accordance with the AME Case Series reporting checklist (available at https://dx.doi.org/10.21037/apm-21-2305).

\section{Methods}

\section{Ethical compliance}

All procedures performed in this study involving human participants were in accordance with the Declaration of Helsinki (as revised in 2013). The study was approved by the ethics committee of the First Affiliated Hospital of Gannan Medical University (No.: 200713104150) and informed consent was taken from all the patients.

\section{Patients}

From 2019 to 2020, we evaluated 6 MFS families in the Heart Medical Centre at the First Affiliated Hospital of Gannan Medical University. The 2010 revised Ghent nosology criteria was used for all evaluations.

\section{Molecular investigation}

In view of the characteristic clinical features of MFS, each family was screened for $8 \mathrm{MFS}$-associated genes (FBN1, TGFBR1, TGFBR2, SMAD3, TGFB2, TGFB3, SKI, and $F B N 2)$ using next-generation sequencing technology (RealOmics Co. Ltd., Shenzhen, China). The average sequencing depth was $150 \times$. After qualifying, removing duplicated reads, aligning and re-calibrating, potential single nucleotide variants and small insertions/deletions were called and filtered using the GATK 3.7 genomic analysis toolkit. The high-confidence variants were noted using SnpEff (Version 4.2: http://snpeff.sourceforge.net/) and were further annotated using the 1000 Genomes Project, Exome Aggregation Consortium (ExAC), Exome Variant Server (EVS), ClinVar, and Online Mendelian Inheritance in Man (OMIM) databases. Pathogenicity interpretation of all variants followed the guidelines for the interpretation of sequence variants of the American College 
of Medical Genetics (13). A variant was considered a causal mutation if it had (I) segregated in a Marfan family, (II) was a de novo mutation if nonsense (i.e., had an in-frame and out-of-frame deletion/insertion, the splice site mutations affected the canonical splice sequence, or it was shown to alter splicing on an mRNA level), (III) was missense and affecting/creating cysteine residues in FBN1, and (IV) was missense affecting conserved residues of the EGF consensus sequence in $F B N 1$ (12). The variants were confirmed by a polymerase chain reaction (PCR) test with oligonucleotide primers and automated Sanger sequencing.

\section{Statistical analysis}

The frequency of each variant in gene targets in the proband was based on a large control dataset from 2,504 individuals in the 1000 Genomes Project phase 5 dataset. Considering MFS is a rare Mendelian disorder, the variants filtered according to minor allele frequency (MAF) thresholds $<0.05 \%$. The functional effect of the identified variants were predicted based on four algorithmic programs including Sorting Intolerant From Tolerant (SIFT) (14), Polymorphism Phenotyping v2 (PolyPhen2) (15), Consensus Deleterious (Condel) (16), and Protein Variation Effect Analyzer (PROVEAN) (17).

\section{Results}

\section{Demographic data and genotype-phenotype analysis of the MFS patient cohort}

We reported the clinical and molecular features of 12 Chinese MFS patients from 6 families in Ganzhou, China. An overview of clinical manifestations observed in the patients are presented in Figure 1. Four of the patients were probands and had a positive family medical history, while the other patients came from families with the sporadic occurrence of MFS (Figure 2). We identified 6 pathogenic mutations, including 1 frameshift, 4 missense affecting/ creating cysteine residues, and 1 nonsense variant. The detailed clinical and molecular features of the patients are summarized in Table 1.

\section{Clinical characteristics}

\section{Family 1}

The proband of this family presented with cardiovascular MFS features, a scoliosis around 150 degrees, and ectopia lentis (EL). The 23-year-old male patient was admitted to our hospital due to chest tightness and shortness of breath. Transthoracic echocardiography revealed mitral anterior and posterior lobe prolapse, profound mitral valve regurgitation, and an enlarged left ventricular end-diastolic diameter (LVEDD) (Figure 3A). Considering the patient's criteria met the formal recommendation for mitral valve replacement surgery, open-heart surgery was performed to replace his mitral valve with a mechanical valve (18). Consistent with the pathogenic features of mitral valve prolapse (MVP), the operative specimen showed that the mitral valve had diffuse irregular thickening and fibrosis. The earliest reliable record of MVP being diagnosed in this patient was 11 years ago, when he was diagnosed with mitral anterior lobe prolapse and mild regurgitation by transthoracic echocardiography. Four years following this initial diagnosis, MVP was also detected in the posterior lobe and severe regurgitation was also present. Remarkably, several severe clinical manifestations were also observed, including a right thoracic scoliosis of 150 degree and EL (Figure 3B). The patient was then diagnosed with MFS because he had EL and his features met the diagnostic criteria (a systemic score $>7$ ). His 9-year-old brother was also diagnosed with MVP and mild mitral valve regurgitation by transthoracic echocardiography while the proband was in hospital. Notably, his 45 -year-old mother had been blind for 10 years and was diagnosed with an isolated case of EL at the age of 20. However, until now she had not displayed any of the typical cardiovascular characteristics of MFS. From the assessment of this family, we found that these 3 affected individuals had shared common symptoms (blurred vision, mild photophobia, and EL) in their ocular systems since childhood. The variant $F B N 1$ c.6331T $>C$ (p.Cys2111Arg) is carried by the proband, his brother, and his mother (Figure 3C).

\section{Families 2-4}

These 3 probands presented with cardiovascular features without a scoliosis. The proband of the second family was a 24-year-old woman who presented to our hospital due to acute chest pain and was diagnosed with a thoracic ascending aortic dissection (TAAD) by computer tomography angiogram (CTA). A year ago, she had undergone aortic root and valve replacement surgery in our hospital. She had also already been suspected of having MFS at the age of 11 years. Her mother also had a vision problem and suddenly died at the age of 30 from aortic dissection. The proband in the $3^{\text {rd }}$ family was a 23 -yearold woman who was diagnosed with severe mitral valve 

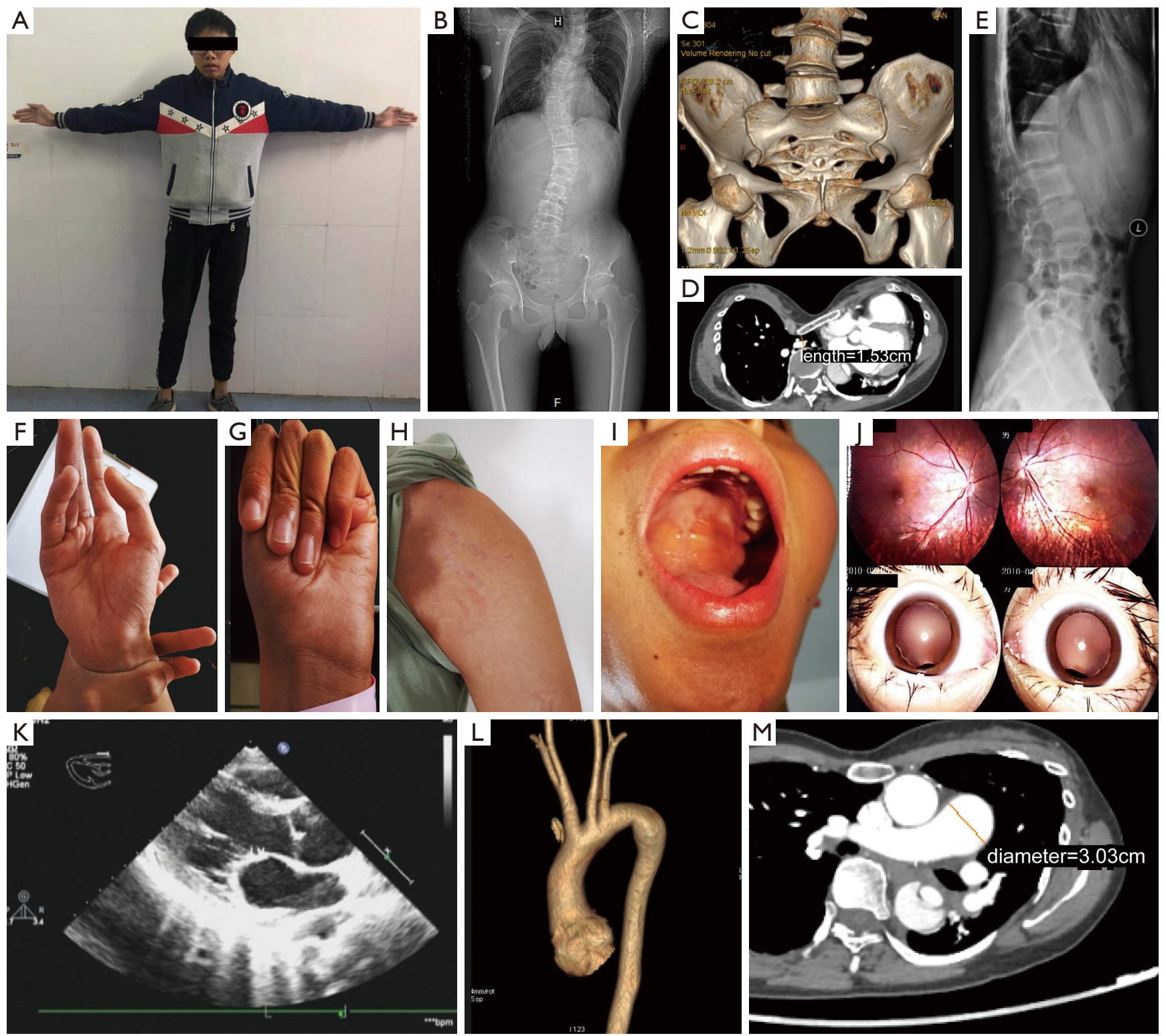

Figure 1 Clinical and instrumental findings observed in the MFS patients. (A) Long arms, legs and fingers; reduced upper segment/lower segment and increased arm span/height without severe scoliosis, (B) curved spine, (C) protrusio acetabulae (D,E) chest sinks, (F) wrist sign, (G) thumb sign, (H) skin striae, (I) retrognathia, (J) ectopia lentis (dislocated lenses), (K) mitral valve prolapse, (L) aortic dilatation at the sinuses of Valsalva, and (M) main pulmonary artery dilatation. This image is published with the patient's consent.

regurgitation, mitral anterior and posterior lobe prolapses, a dilated descending aorta, enlarged LVEDD, and poor left ventricular ejection fraction (LVEF: $55 \%$ ) by transthoracic echocardiography. The patient's father died at the age of 29 from a cardiac event. The proband in the $4^{\text {th }}$ family was a sporadic case in an 18-year-old man who presented with modest mitral valve regurgitation caused by mitral lobe prolapse, as well as a dilated aortic root.

\section{Families 5-6}

These 2 probands presented cardiovascular features and a scoliosis. Both had comprehensive skeletal manifestations, such as progressive scoliosis, pectus excavatum, protrusio acetabuli, and reduced elbow extension. Furthermore, they had typical MFS facial characteristics, including dolichocephaly, malar hypoplasia, enophthalmos, retrognathia, and down-slanting palpebral fissures, but no 


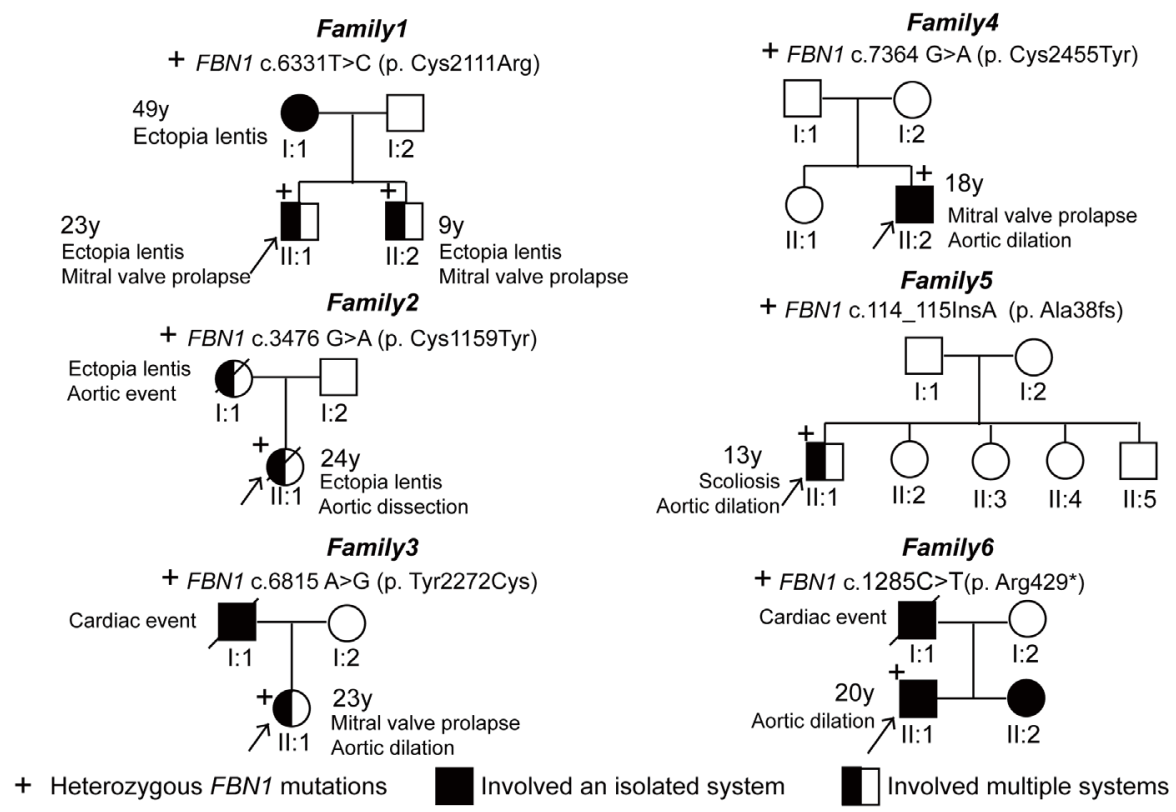

Figure 2 Family history and the clinical and molecular characteristics of all families in the present study.

EL. Regarding their cardiovascular systems, they displayed dilatation of the aortic root and pulmonary artery, but no MVP (Table 1). As the 2 patients had a dilated aortic root and their features met the diagnostic criteria (a systemic score $>7$ ), they were both diagnosed with MFS. Notably, the proband in the 5th family, a 13-year-old boy, was treated with incisional hernias at the age of 2 , and showed stretch marks at the age of 11 (Figure 3D). The proband in the $6^{\text {th }}$ family, a 21-year-old boy, had aortic arch dysplasia branches characterized by a common brachiocephalic trunk and common left carotid arteries. In addition, at the age of 10 , stretch marks and multiple bullae were observed in the upper lobe of both lungs. His father resembled the proband in looks and died by aortic dissection at 30. His 16-year-old sister also had a dilated aortic root (Figure 3E).

\section{Mutation identification and bioinformatic analysis}

In the studied families, we identified 4 heterozygous missense, 1 heterozygous nonsense, and 1 heterozygous insertion mutations of FBN1. This included 1 novel mutation (c.114_115ins $A$ ), as well as 5 known variants (c.6331T>C,c.3476G>A,c.6815A>G,c.7364G>A and c.1285C $>T$ ). The novel mutation had not been noted in published articles or existed in known databases [Cardiomyopathy Database (CMDB), ClinVar, the Human
Gene Mutation Database (HGMD), and UniProt]. Of the missense mutations, they all involved a cysteine, including 3 replacing and 1 creating cysteines. Except for $c .6331 T>C$, which replaced a cysteine in the TB-like domain, the remaining missense mutations altered cysteine residues in the calcium binding EGF (cbEGF)-like domains. These affected residues were revealed to be evolutionary conserved by the MutationTaster application (19). Furthermore, the changes probably led to the incorrect pairing of cysteine residue and had a predictable detrimental effect on both the domain itself and the neighboring cbEGF-like domains. The novel insertion mutation changed the ribosome reading frame and caused premature termination of translation. The variant $c .114 \_115$ ins $A$ was a frameshift mutation in exon 2 of FBN1. The insertion created a change from amino acids 39 to 54 and a deletion of big fragments from amino acids 55 to 2871 . This deleterious effect on protein function was also revealed by MutationTaster. c.1285C $>T$ was a nonsense mutation leading to premature translation termination at amino acid position 429 .

\section{Genotype-phenotype correlation}

The 6 pathogenic mutations were divided into 2 mutation categories: HI mutations and DN mutations. A range of qualitative parameters involving skeletal, cardiovascular, 


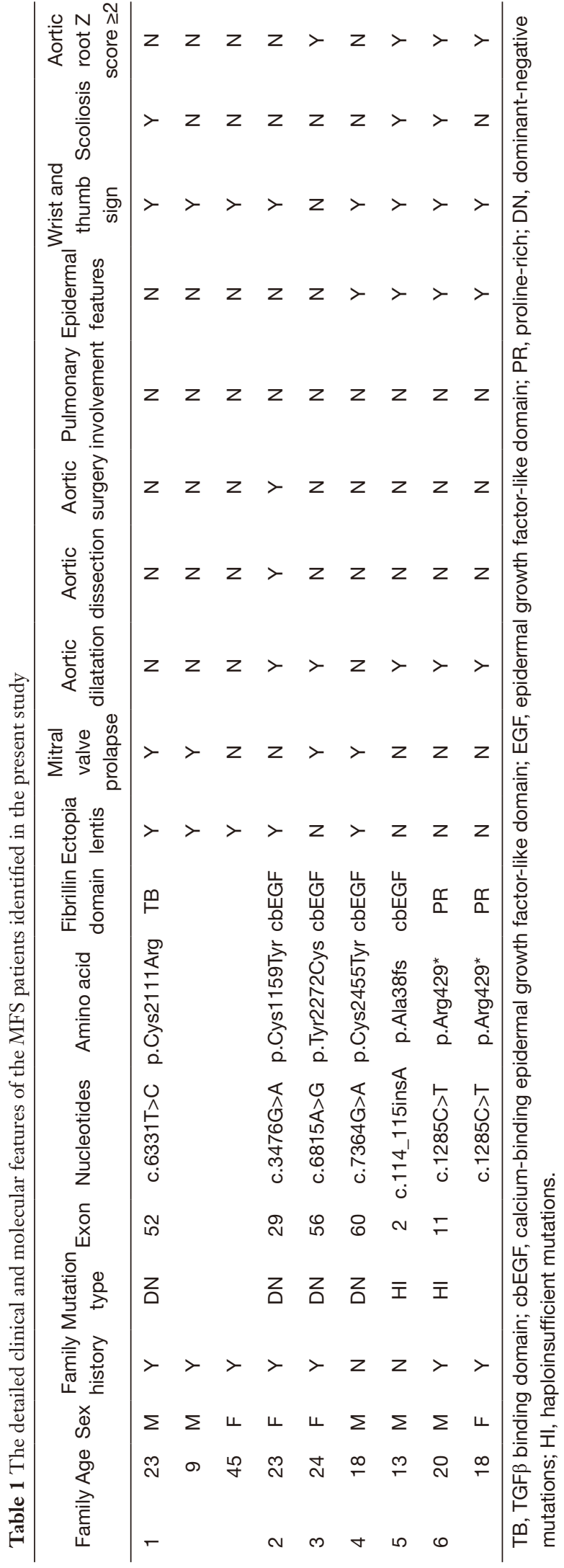

and ophthalmologic systems were compared between the probands for the 2 types of mutations in FBN1. Several skeletal features were shared by all affected individuals including arachnodactyly, wrist and thumb sign, and wrist joint hypermobility. Five of the 6 probands $(83.3 \%)$ were observed to have aortic dilation or dissection when they were diagnosed with MFS. Compared with the patients with HI mutations, MVP and EL were uniquely found in the patients where DN mutations substituted or produced a cysteine. Two probands with FBN1 HI mutations displayed typical facial features of MFS.

\section{Discussion}

FBN1 pathogenic mutations have been linked with a wide range of phenotypic variabilities now often called "type1 fibrillinopathies". These variabilities range from single organ involvement, such as isolated EL and isolated ascending aortic aneurysm and dissection, to multiorgan dysfunction syndrome, like typical MFS and lethal neonatal MFS. Several systems and organs are affected by FBN1 mutations, including the lungs, skin, ocular, cardiovascular, and skeletal systems. Most MFS patients ultimately develop severe cardiovascular outcomes, including aortic dilatation, MVP, and left ventricular dysfunction, etc. In the present study, we analyzed the data of 6 families who had molecular variants of MFS, and who met the clinical criteria for MFS diagnosis. The distinction of phenotype characteristics between their different mutation types were then observed.

As in other studies, a high percentage of mutations carried by the MFS probands of our study involved cysteine residues [4/6 (67\%)] (20,21). In 3 of 4 cases, these residues were located within cbEGF-like domains, while the remaining 1 was in the TB-like domain. Notably, the clinical manifestations of individuals with an identical $F B N 1$ varied widely with respect to organ-system involvement and severity. For example, in family 1 , the proband had significant cardiovascular, skeletal, and ocular problems, although his mother presented only with isolated EL. Coupled with detailed clinical descriptions in previous studies, we also accumulated more mutation data to observe the variability of the mutations. The proband with c.6815A>G in this study had severe MVP without EL, but in a previous report, a patient with the identical mutation was diagnosed with isolated EL without any cardiovascular problems (22). The variability on tissue distribution, ranging from typical MFS to isolated MVP, were also observed in the individuals with $c .7364 G>A$ (21). As a result, it is worth 


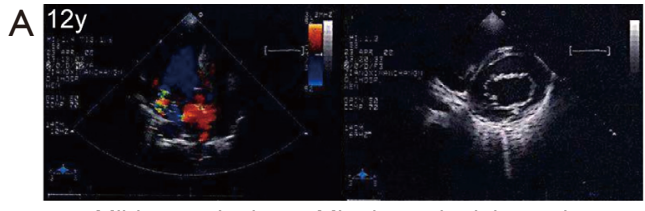

Mild regurgitation Mitral anterior lobe prolapse

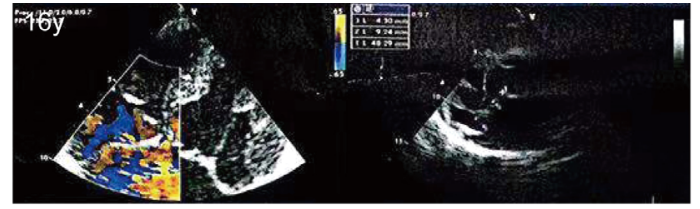

Severe regurgitation
Mitral anterior and posterior lobe prolapse

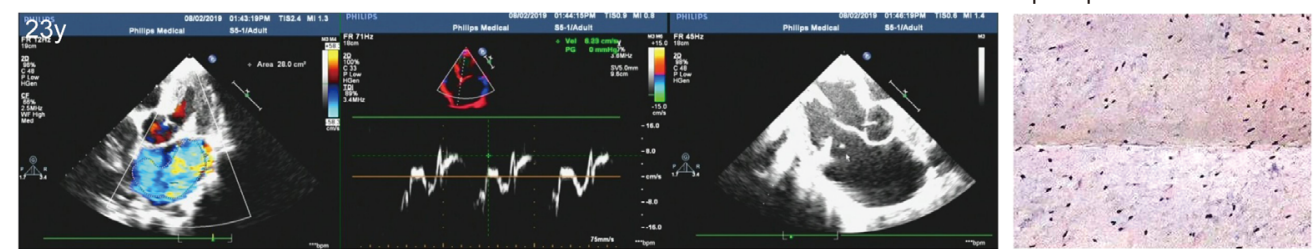

Profoundly regurgitation Left atrioventricular Mitral anterior and posterior enlargement lobe prolapse with calcification and fibrosis

B Family one II:1

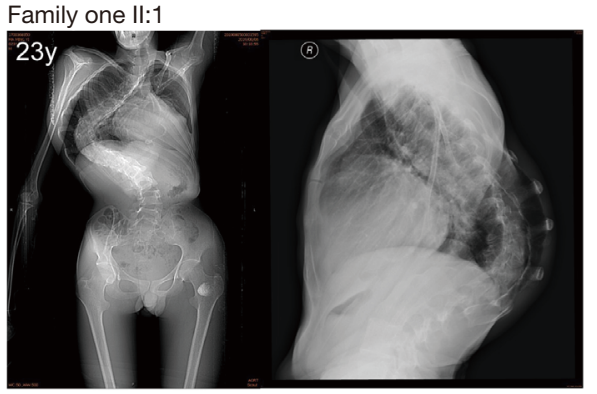

D Family five

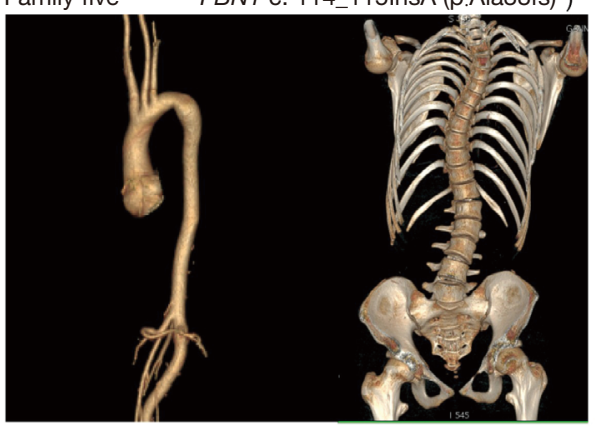

I:1, I:2, II:2, II:3, II:4, II:5

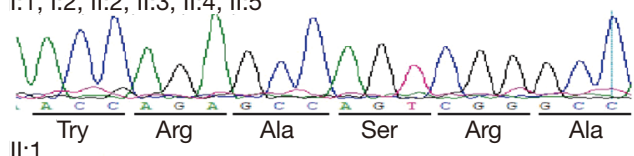
II:1
C Family one I:1, II:1, II:2

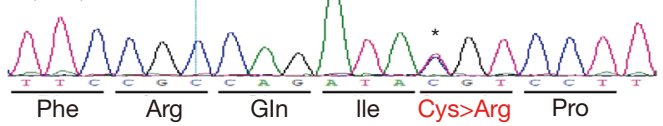

l:2

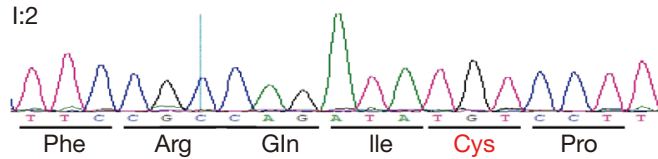

E Family six $\quad$ FBN1 c. $1285 \mathrm{C}>\mathrm{T}\left(\mathrm{p} . \operatorname{Arg} 429^{*}\right)$

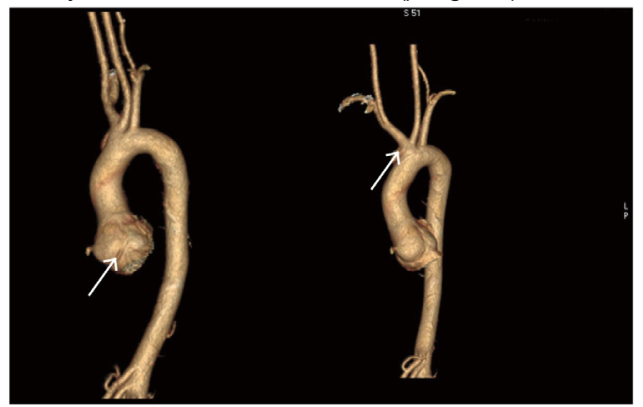

$1: 2$
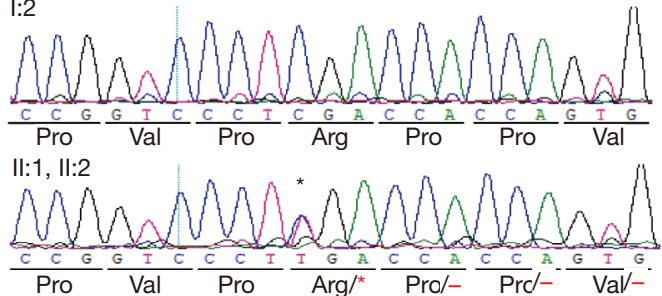

Figure 3 The clinical and molecular characteristics of families 1, 5 and 6. (A) Natural history of mitral valve prolapse of the proband (II:1) in family 1 between age of 12 and 23, sections of surgically excised mitral valve leaflet were paraffin-embedded, stained with hematoxylin and eosin for light microscopy (400x), and (B) his thoracic vertebral features. (C) Sanger sequencing showing the variant FBN1 c.6331T>C (p.Cys2111Arg) is carried by I:1, II:1, and II:2 in family 1 (indicated by the asterisk), and the corresponding sequencing of the unaffected family member. (D,E) Thoracic aortic features of the proband (II:1) in families 5 and 6. Sanger sequencing showing the FBN1 variants c.114_115insA (p.Ala38fs) and c.1285C>T (p.Arg429*) carried by families 5 and $6 .{ }^{*}$, pathogenic mutations. White arrows indicate the location of the lesion. MFS, Marfan syndrome. 
noting that the tissue distribution characteristics of the cysteine mutants might be relative to whether MVP and EL are present alone or in combination. The strong correlation between the presence of a mutation affecting a cysteine residue and both EL and MVP in this study is consistent with the conclusions of other reports $(23,24)$. As the most important element in the FBN1 modular, cysteine residues in both EGF and TB-like motifs have proven to be critical to both the function of the domain itself and neighboring domains. Consequently, these residues are responsible for maintaining microfibrils in an ordered arrangement $(25,26)$. The cbEGF-like motif has 6 conserved cysteine residues that form 3 conserved disulfide bonds in a $1-3,2-4,5-6$ arrangement to maintain structural integrity. The TB motif has 8 highly conserved cysteine residues that function to hold TB in an inactive complex. Moreover, mutations in exons 24-32 are universally acknowledged to be associated with neonatal MFS and a more severe prognosis in nonneonatal MFS, including higher probability of aortic dilatation, aortic surgery, and a shorter survival time $(24,27)$. In this study, the patient with $c .3476 G>A$ was the only one found to have mutations in exons $23-32$. She also underwent TAAD twice in 2 years in her 20s.

Unlike the patients with cysteine-related mutants, the 3 individuals with $\mathrm{HI}$ mutations in this study did not present with EL or MVP. Six other studies have drawn conclusions consistent with this finding, suggesting that when compared with HI mutations, a significantly higher frequency of EL is associated with cysteine substitutions $(5,21,23,24,28,29)$. In this study, we also observed that the individual with a big deletion in FBN1 had a more serious skeletal and skin character than the patients with a small deletion, partly indicating the important effect $F B N 1$ has on a patient's skeleton and skin. An international study supports this observation. They found that patients with a HI mutation had a more severe skeletal and skin phenotype than patients with a DN mutation (24). Furthermore, recent studies on phenotype-genotype correlations in MFS patients also suggest a connection between HI mutations and aortic events. Baudhuin et al. first reported that HI mutations are associated with a higher percentage of aortic events and a lower presentation age (30). Their findings were supported by the data of 2 cohort studies in Chinese and Mediterranean MFS patients (12,31). This worse prognosis was also confirmed by a large prospective study of 570 MFS patients, in which $\mathrm{HI}$ mutation carriers were found to have a 2.4 times higher risk of requiring aortic dissection (10).
Furthermore, patients with HI mutations had lower aortic root growth rates and were noted to benefit more from treatment with Losartan (32).

The association between FBN1 mutation types and clinical divergent manifestations might be because of its tissue-specific nature of fibrillin-1 assemblies. The fibrillar structure have been proven to govern the regulatory activity on resident cells and signal molecule in each tissue (33). For example, fibrillin-1 impacted local TGF $\beta$ activity in the thoracic aortic aneurysm, but not found to be a determinant of dilated cardiomyopathy development (34). Moreover, it is worth mentioning that patients with isolated EL was categorically diagnosed with MFS according to FBN1 mutations, indicating that the individual changes of fibrillinopathies with MFS (35). As a result, a genetic testing was initiated as a determinant for etiological diagnosis by the sequence analysis of FBN1, TGFBR1, TGFBR2, etc. as described in the method, currently further informed risk stratification, the strategy of surveillance and treatment, and a prenatal testing at pregnancy. As the top mortality cause for MFS patients, the HI carriers were more predisposed to have thoracic aortic dissection, meanwhile the missense mutants had a smaller risk of the rupture of aortic aneurysm except for those in exon 24-32 (30,31). Noteworthy, FBN1 mutations also largely contribute to pathogenesis of sporadic nonsyndromic aortic dissection, the ones were younger in the onset aortic events than non-FBN1-mutation aortic dissection patients (36). Interestingly, the difference of the mutation locations was not identified between the nonsyndromic and syndromic patients (36).

To conclude, in this study we described the clinical manifestations and FBN1 mutations of 6 MFS families in China, adding one novel variant to the existing spectrum of FBN1 mutations. Furthermore, we demonstrated the connection between different mutation types and clinical manifestations. Although there was considerable variability in the onset of age, tissue distribution, and severity of clinical manifestations both among and between the affected families, the high proportion of MFS patients observed in our study supports previous studies which have focused on the correlation between clinical manifestations and mutation types in MFS. By furthering both the clinical and basic research on the FBN1 mutation in MFS patients, this study is beneficial to prognostic implications and can help facilitate early diagnosis and symptomatic treatment of the disease, preventing unnecessary interventions and 
adverse outcomes.

\section{Acknowledgments}

The authors wish to thank all patients for their cooperation during the diagnosis process.

Funding: This work was financially supported by the National Natural Science Foundation of China (31671288; 81960326), Science and Technology Project of Jiangxi Health Committee (202130676), Primary Research \& Development Plan of Jiangsu Province (20192BBG70039), and Innovation Team Foundation (TD201902).

\section{Footnote}

Reporting Checklist: The authors have completed the AME Case Series reporting checklist. Available at https://dx.doi. org/10.21037/apm-21-2305

Data Sharing Statement: Available at https://dx.doi. org/10.21037/apm-21-2305

Conflicts of Interest: All authors have completed the ICMJE uniform disclosure form (available at https://dx.doi. org/10.21037/apm-21-2305). The authors have no conflicts of interest to declare.

Ethical Statement: The authors are accountable for all aspects of the work in ensuring that questions related to the accuracy or integrity of any part of the work are appropriately investigated and resolved. All procedures performed in this study involving human participants were in accordance with the Declaration of Helsinki (as revised in 2013). The study was approved by the ethics committee of the First Affiliated Hospital of Gannan Medical University (No.: 200713104150) and informed consent was taken from all the patients.

Open Access Statement: This is an Open Access article distributed in accordance with the Creative Commons Attribution-NonCommercial-NoDerivs 4.0 International License (CC BY-NC-ND 4.0), which permits the noncommercial replication and distribution of the article with the strict proviso that no changes or edits are made and the original work is properly cited (including links to both the formal publication through the relevant DOI and the license). See: https://creativecommons.org/licenses/by-nc-nd/4.0/.

\section{References}

1. Pyeritz RE, McKusick VA. The Marfan syndrome: diagnosis and management. N Engl J Med 1979;300:772-7.

2. Pólos M, Benke K, Ágg B, et al. Psychological factors affecting Marfan syndrome patients with or without cardiac surgery. Ann Palliat Med 2020;9:3007-17.

3. Loeys BL, Dietz HC, Braverman AC, et al. The revised Ghent nosology for the Marfan syndrome. J Med Genet 2010;47:476-85.

4. Dietz HC, Cutting GR, Pyeritz RE, et al. Marfan syndrome caused by a recurrent de novo missense mutation in the fibrillin gene. Nature 1991;352:337-9.

5. Arbustini E, Grasso M, Ansaldi S, et al. Identification of sixty-two novel and twelve known FBN1 mutations in eighty-one unrelated probands with Marfan syndrome and other fibrillinopathies. Hum Mutat 2005;26:494.

6. Saharinen J, Keski-Oja J. Specific sequence motif of 8-Cys repeats of TGF-beta binding proteins, LTBPs, creates a hydrophobic interaction surface for binding of small latent TGF-beta. Mol Biol Cell 2000;11:2691-704.

7. Verstraeten A, Alaerts M, Van Laer L, et al. Marfan Syndrome and Related Disorders: 25 Years of Gene Discovery. Hum Mutat 2016;37:524-31.

8. Gray JR, Bridges AB, Faed MJ, et al. Ascertainment and severity of Marfan syndrome in a Scottish population. J Med Genet 1994;31:51-4.

9. Collod-Béroud G, Le Bourdelles S, Ades L, et al. Update of the UMD-FBN1 mutation database and creation of an FBN1 polymorphism database. Hum Mutat 2003;22:199-208.

10. Franken R, Groenink M, de Waard V, et al. Genotype impacts survival in Marfan syndrome. Eur Heart J 2016;37:3285-90.

11. Faivre L, Collod-Beroud G, Callewaert B, et al. Clinical and mutation-type analysis from an international series of 198 probands with a pathogenic FBN1 exons 24-32 mutation. Eur J Hum Genet 2009;17:491-501.

12. Becerra-Muñoz VM, Gómez-Doblas JJ, Porras-Martín C, et al. The importance of genotype-phenotype correlation in the clinical management of Marfan syndrome. Orphanet J Rare Dis 2018;13:16.

13. Richards S, Aziz N, Bale S, et al. Standards and guidelines for the interpretation of sequence variants: a joint consensus recommendation of the American College of Medical Genetics and Genomics and the Association for 
Molecular Pathology. Genet Med 2015;17:405-24.

14. Vaser R, Adusumalli S, Leng SN, et al. SIFT missense predictions for genomes. Nat Protoc 2016;11:1-9.

15. Adzhubei I, Jordan DM, Sunyaev SR. Predicting functional effect of human missense mutations using PolyPhen-2. Curr Protoc Hum Genet 2013; Chapter 7:Unit7.20.

16. González-Pérez A, López-Bigas N. Improving the assessment of the outcome of nonsynonymous SNVs with a consensus deleteriousness score, Condel. Am J Hum Genet 2011;88:440-9.

17. Choi Y, Chan AP. PROVEAN web server: a tool to predict the functional effect of amino acid substitutions and indels. Bioinformatics 2015;31:2745-7.

18. Nishimura RA, Otto CM, Bonow RO, et al. 2014 AHA/ ACC guideline for the management of patients with valvular heart disease: a report of the American College of Cardiology/American Heart Association Task Force on Practice Guidelines. J Thorac Cardiovasc Surg 2014;148:e1-e132.

19. Schwarz JM, Cooper DN, Schuelke M, et al. Mutation Taster2: mutation prediction for the deepsequencing age. Nat Methods 2014;11:361-2.

20. Mannucci L, Luciano S, Salehi LB, et al. Mutation analysis of the FBN1 gene in a cohort of patients with Marfan Syndrome: A 10-year single center experience. Clin Chim Acta 2020;501:154-64.

21. Hernándiz A, Zúñiga A, Valera F, et al. Genotype FBN1/ phenotype relationship in a cohort of patients with Marfan syndrome. Clin Genet 2021;99:269-80.

22. Comeglio P, Johnson P, Arno G, et al. The importance of mutation detection in Marfan syndrome and Marfanrelated disorders: report of $193 \mathrm{FBN} 1$ mutations. Hum Mutat 2007;28:928.

23. Rommel K, Karck M, Haverich A, et al. Identification of 29 novel and nine recurrent fibrillin-1 (FBN1) mutations and genotype-phenotype correlations in 76 patients with Marfan syndrome. Hum Mutat 2005;26:529-39.

24. Faivre L, Collod-Beroud G, Loeys BL, et al. Effect of mutation type and location on clinical outcome in 1,013 probands with Marfan syndrome or related phenotypes and FBN1 mutations: an international study. Am J Hu Genet 2007;81:454-66.

25. Dietz HC, Saraiva JM, Pyeritz RE, et al. Clustering of fibrillin (FBN1) missense mutations in Marfan syndrome patients at cysteine residues in EGF-like domains. Hum Mutat 1992;1:366-74.

26. Jin C, Yao K, Jiang J, et al. Novel FBN1 mutations associated with predominant ectopia lentis and marfanoid habitus in Chinese patients. Mol Vis 2007;13:1280-4.

27. Lönnqvist L, Karttunen L, Rantamäki T, et al. A point mutation creating an extra $\mathrm{N}$-glycosylation site in fibrillin-1 results in neonatal Marfan syndrome. Genomics 1996;36:468-75.

28. Schrijver I, Liu W, Odom R, et al. Premature termination mutations in FBN1: distinct effects on differential allelic expression and on protein and clinical phenotypes. Am J Hum Genet 2002;71:223-37.

29. Biggin A, Holman K, Brett M, et al. Detection of thirty novel FBN1 mutations in patients with Marfan syndrome or a related fibrillinopathy. Hum Mutat 2004;23:99.

30. Baudhuin LM, Kotzer KE, Lagerstedt SA. Increased frequency of FBN1 truncating and splicing variants in Marfan syndrome patients with aortic events. Genet Med 2015;17:177-87.

31. Xu S, Li L, Fu Y, et al. Increased frequency of FBN1 frameshift and nonsense mutations in Marfan syndrome patients with aortic dissection. Mol Genet Genomic Med 2020;8:e1041.

32. Franken R, den Hartog AW, Radonic T, et al. Beneficial Outcome of Losartan Therapy Depends on Type of FBN1 Mutation in Marfan Syndrome. Circ Cardiovasc Genet 2015;8:383-8.

33. Ramirez F. Introduction to the mini-review series "Extracellular determinants of cell signaling". Matrix Biol 2015;47:1-2.

34. Ramirez F, Caescu C, Wondimu E, et al. Marfan syndrome; A connective tissue disease at the crossroads of mechanotransduction, TGF $\beta$ signaling and cell stemness. Matrix Biol 2018;71-72:82-9.

35. Chandra A, Patel D, Aragon-Martin JA, et al. The revised ghent nosology; reclassifying isolated ectopia lentis. Clin Genet 2015;87:284-7.

36. Tan L, Li Z, Zhou C, et al. FBN1 mutations largely contribute to sporadic non-syndromic aortic dissection. Hum Mol Genet 2017;26:4814-22.

(English Language Editor: J. Goetz)

Cite this article as: Duan Y, Li P, Ding T, Wang Y, Liao Y, Du Z, Ling J, Liu S, Li W, Liu Z. Combining clinical examination with exome sequencing for the diagnosis and treatment of Marfan syndrome: a case series of 6 families from China. Ann Palliat Med 2021;10(9):9953-9962. doi: 10.21037/apm-21-2305 